\section{Orientation of erythrocytes in optical trap revealed by confocal fluorescence microscopy}

\author{
Khyati Mohanty, ${ }^{a, b}$ Samarendra Mohanty, ${ }^{b,}{ }^{*}$ \\ Shamci Monajembashi, ${ }^{\mathrm{C}}$ and Karl Otto Greulich ${ }^{\mathrm{c}}$ \\ a University of California, Department of Biomedical \\ Engineering, 305 Rockwell Engineering Center, Irvine, \\ California 92697 \\ ${ }^{\mathrm{b}}$ University of California, Beckman Laser Institute, 1002 \\ Health Science Road, Irvine, California 92612 \\ ${ }^{\mathrm{C}}$ Fritz Lipmann Institute, Leibniz Institute for Age Research, \\ Department of Single Cell and Single Molecule \\ Techniques, Beutenbergstrasse 11, D-07745 Jena, Germany
}

\begin{abstract}
There has been considerable current interest in the rotational behavior of red blood cells (RBCs) in optical tweezers. However, the mechanism of rotation in polarized tweezers is still not well understood and conflicts exist in the understanding of this phenomenon. Therefore, we reexamined the underlying phenomenon by use of confocal fluorescence microscopy in combination with optical tweezers. Under different osmolarities of the buffer, the three-dimensionally reconstructed images showed that the trapped RBC maintains its shape and is oriented in the vertical direction. Using dual optical tweezers, the RBC could also be oriented three-dimensionally in a controlled manner. The mechanism of orientation and alignment of RBCs with the polarization of the tweezers' beam was attributed to its form-birefringence rather than optical birefringence. $\odot 2007$ Society of Photo-Optical Instrumentation Engineers. [DOI: 10.1117/1.2822365]
\end{abstract}

Keywords: optical tweezers; polarization; rotation; red blood cell; confocal microscopy.

Paper 07174LR received May 15, 2007; revised manuscript received Oct. 2, 2007; accepted for publication Oct. 7, 2007; published online Dec. 28, 2007.

There is considerable interest in optically controlled orientation or rotation of microscopic objects for applications in microfluidics and bionanotechnology. It has been shown ${ }^{1}$ that the normal red blood cell (RBC), in hypertonic buffer, is a naturally occurring micromotor thats rotates by itself when placed in a trap laser beam. Recently, we have reported ${ }^{2}$ the use of such an optically driven RBC-motor for microfluidic applications like control of flow of fluids, although nonliving rotors ${ }^{3}$ will be more useful in a microfluidic environment. Since in a fixed laser power, the speed of tweezers-actuated $\mathrm{RBC}$-motors depends on the viscosity of the surrounding medium, the motor can also be used for microrheology of fluids. Further, since discotic RBCs are aligned with the direction of polarization, ${ }^{2}$ very small fluid flow can overcome the polarization-induced alignment at lower trapping beam powers and thus can be used as a microfluidic flow rate sensor. In a circularly polarized optical trap, Dharmadhikari et al. reported $^{4}$ trap-induced folding of the $\mathrm{RBC}$, leading to optical

*Tel: (949) 824-7859; E-mail: smohanty@uci.edu birefringence and its use as a naturally occurring microrotor. However, theoretical considerations indicate that erythrocytes cannot be folded at the low trapping beam power $(\sim 10 \mathrm{~mW})$ used by Dharmadhikari et al. At this power, the force exerted into the direction of beam propagation is few $\mathrm{pN}$, and only a fraction of this value can be exerted in any direction perpendicular to this. ${ }^{5}$ This is the force that can stretch a single DNA molecule but probably cannot deform a whole cell membrane. Further, to fold an RBC, one requires a pivot point around which the two diametrically opposite ends of the discotic RBC can be folded, as demonstrated using three tweezers (see, for example, Ref. 5). This required a higher trapping beam power than that of Dharmadhikari et al. ${ }^{4}$ and a relaxation (or unfolding) time an order of magnitude shorter than the earlier report. ${ }^{4}$ Also, it is quite natural that the $\mathrm{RBC}$ in conventional optical tweezers would like to orient in edge-on manner rather than fold, as folding requires more energy and a pivoting line or point. Therefore, in the present work, we reexamine the orientational behavior of erythrocytes in an optical trap. We report the use of confocal fluorescence microscopy and dual optical tweezers to determine change in shape of the RBC (if any) under radiation pressure. Coinciding with our earlier report, ${ }^{6}$ no folding of the RBC to the rod shape was observed, thus ruling out the suggested ${ }^{4}$ induction of optical birefringence. When optically trapped, the discotic $\mathrm{RBC}$ was rather found to orient with its plane along the direction of the trapping beam.

RBCs from fresh blood were suspended in phosphate buffered saline (PBS) at different osmolarities. For confocal microscopic imaging, the RBCs were stained with the membrane-potential-sensitive dye DIOC5 (3, $3^{\prime}$ dipentyloxacarbocyanine iodide) at a final concentration of $20 \mu \mathrm{M}$. The fluorescence was excited using an Ar-Ion laser line of $488 \mathrm{~nm}$ (power: $2 \mathrm{~mW}$ ), and fluorescence was collected using a long-pass filter. The experimental setup $(\text { details })^{7}$ consists of an inverted confocal laser scanning microscope (LSM 510, Carl Zeiss GmbH, Jena, Germany) equipped with an argon-ion laser and a green $\mathrm{HeNe}$ laser $(543 \mathrm{~nm})$ as scanning lasers. The LSM 510 carries out $z$-sectioning for three-dimensional (3-D) imaging by moving the objective along the $z$ direction in order to scan optical sections of an object at different $z$ positions. For trapping, the cw output of an Nd: YAG laser (1064 nm; Spectra Physics, Darmstadt, Germany) operating in fundamental Gaussian mode was coupled to the $100 \times$ Plan Neofluor phase objective of the microscope through its epifluorescence port. A singlemode fiber (BTO Bungert GmbH, Germany) directs the laser light from the Nd: YAG laser to the rear side of the microscope. The fiber output efficiency with the single-mode fiber coupler used (Thorlabs, Grünberg, Germany) is about $60 \%$. A convex lens $(f=40 \mathrm{~mm})$, added externally into the path of the laser beam, focuses the light emerging from the fiber intothe epifluorescence illumination path, where another convex lens $(f=150 \mathrm{~mm})$ is located. The single-mode fiber together with the external lens presents a suitable beam expander, because of the diverging beam emerging from the fiber. Finally, the dichroic mirrors located in the fluorescence reflector slide direct the laser light to the back aperture of the objective that has to be entirely illuminated by the infrared laser light to

1083-3668/2007/12(6)/060506/3/\$25.00 @ 2007 SPIE 


\section{JBO LETTERS}
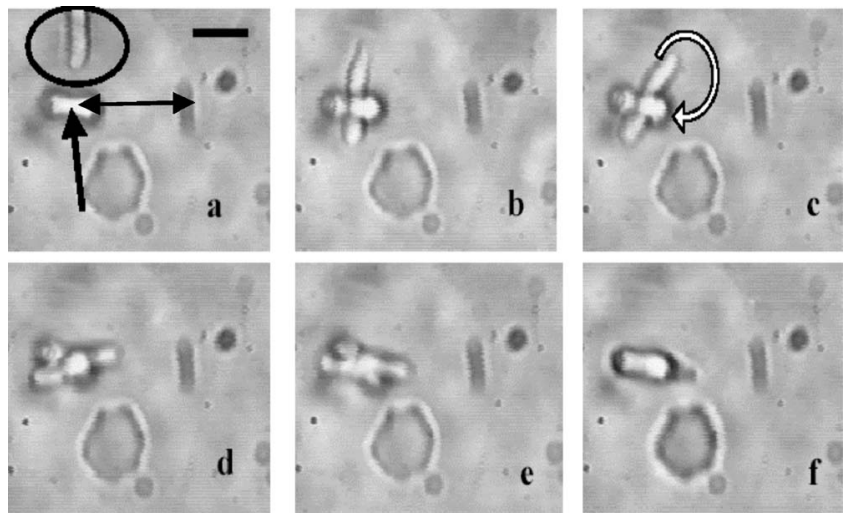

Fig. 1 Time-lapse (400 ms) images of alignment of multiple RBCs in linearly polarized optical tweezers. All images are in same magnification. Scale bar: $5 \mu \mathrm{m}$.

obtain an almost diffraction-limited beam for good trapping efficiency. For the use of the optical tweezers in laser scanning mode, another dielectric mirror (Laser Optik GmbH, Garbsen, Germany) is placed on the laser scanning position of the fluorescence reflector slide that otherwise would be empty. It reflects the infrared laser light and is simultaneously transparent for the scanning lasers coming from below and the emitted fluorescence. Hence, the complete imaging capabilities of the original LSM 510 are maintained. For generating dual tweezers, the infrared laser beam was split into two beams on a time-sharing basis by an acousto-optic deflector. The relative focal positioning of the two trapping beams was adjusted by changing the angle and divergence of the beams as they enter the objective back aperture.

We show in Fig. 1 the digitized video images of the alignment of multiple RBCs in linearly polarized optical tweezers. The arrow-marked RBC is trapped (a) and gets aligned along the direction of polarization. Another vertically oriented RBC gets trapped when it reaches close to the trapped $\mathrm{RBC}$ and slowly rotates clockwise [(b) to (d)] to align along the polarization direction (marked by a double-headed arrow) of the linearly polarized optical tweezers [panel (e)]. The two RBCs were found to align one above the other in the vertical direction (f). By relative focal positioning of two optical tweezers ( 1 and 2, each having power of $\sim 20 \mathrm{~mW}$ ), the discotic RBC (in $300 \mathrm{mOsm} / \mathrm{kg}$ solution) trapped at two diametrically opposite ends, could also be oriented three-dimensionally in a controlled manner, as shown in Fig. 2. Since no folding of the $\mathrm{RBC}$ was observed under the optical trapping beam, the rotational mechanism based on optical birefringence caused by folding of RBC as suggested earlier ${ }^{4}$ can be ruled out. In hypotonic buffer $(\sim 100 \mathrm{mOsm} / \mathrm{Kg})$, the trapped RBC maintains its circular cross section in both the horizontal and vertical planes, leading to a three-dimensionally spherical shape (data not shown). In isotonic buffer $(300 \mathrm{mOsm} / \mathrm{Kg})$, the horizontal cross section of the trapped RBC was found to be elliptical and the vertical cross section as circular. The threedimensionally reconstructed image [Figs. 3(a) to 3(c)] shows that the trapped RBC (arrow-marked) maintains its discotic shape while being oriented in the vertical direction. In hypertonic buffer $(600 \mathrm{mOsm} / \mathrm{Kg})$, the reconstructed image of the RBCs under three viewing projections [Figs. 3(d) to 3(f)] shows that all of the RBCs are asymmetrically deformed by
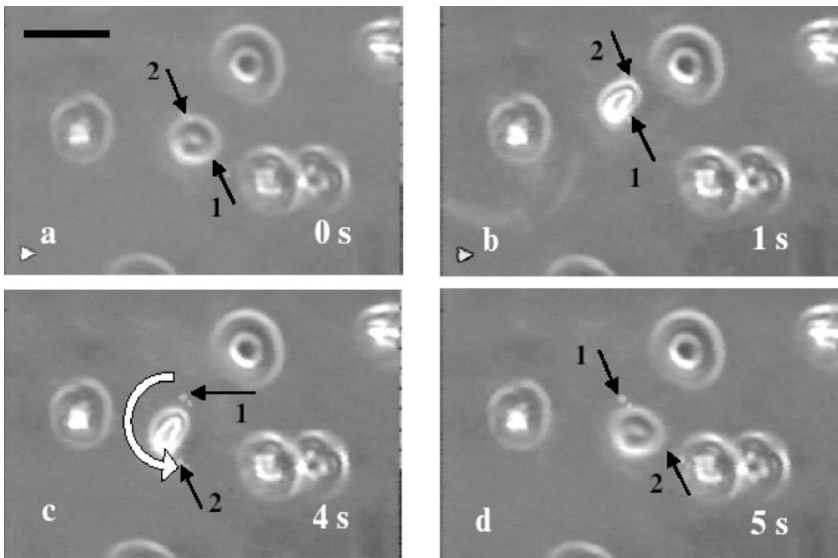

Fig. 2 Three-dimensional (3-D) reorientation of RBC using dual optical tweezers. The discotic RBC trapped at two diametrically opposite ends (arrow marked) is being tilted from horizontal (a) to a vertical orientation (b). By rotation of one of the tweezers, the vertically tilted RBC could be rotated in anticlockwise direction (c). The RBC could be returned back to the horizontal plane $(d)$ by repositioning of one of the tweezers. All images are in same magnification. Scale bar: $10 \mu \mathrm{m}$.

the osmolarity of the buffer and the trapped RBC is vertically oriented. Further, under both isotonic and hypertonic situations (Fig. 3), the vertically oriented RBC is aligned along the plane of polarization of the trapping beam.

Like optically birefringent particles, ${ }^{8}$ there is an alignment (and rotation) effect of disk-like particles (lacking spherical
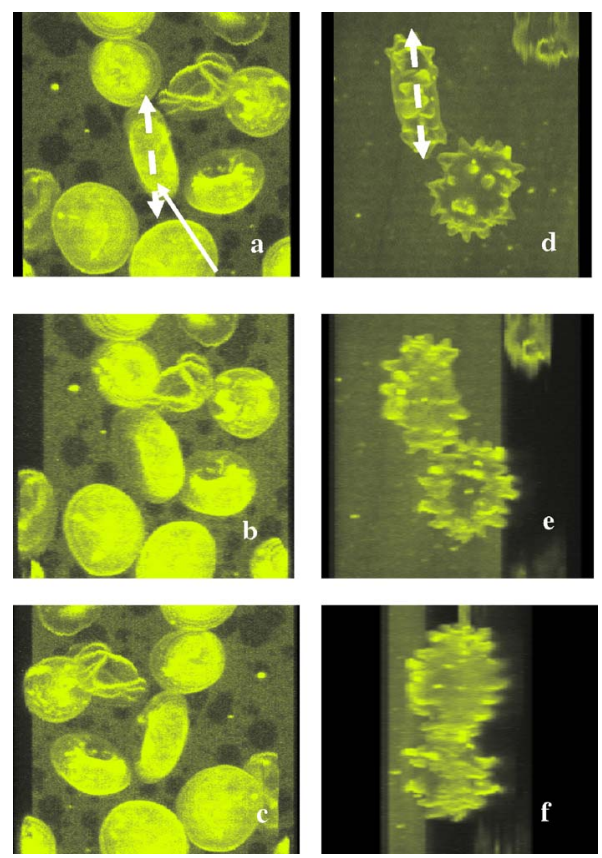

Fig. 3 Confocal microscopic images of RBCs in an isotonic [(a) to (c), Video 1] and a hypertonic [(d) to (f), Video 2] buffer, with the arrow-marked areas exposed to an optical trap beam (with power $\sim 120 \mathrm{~mW}$ ) in three different viewing projections. The faint fluorescence background on the surface of the coverslip can be seen in all the panels. The plane of polarization of the trap beam is shown by the double-headed arrow. (Video 1, MPEG, $834 \mathrm{~KB}$ [URL: http://dx.doi.org/10.1117/1.2822365.1]; Video 2, AVI, 53.2 MB [URL: http://dx.doi.org/10.1117/1.2822365.2].) 


\section{JBO LETTERS}

symmetry) with polarization due to their form-birefringence. Since by rotating the polarization of the trapping beam, the discotic RBC can be rotated, the orienting torque is believed to originate from the anisotropic scattering of polarized light by the trapped RBC. Indeed, orientation of objects (having form-birefringence) by rotating the plane of polarization of the linearly polarized trapping beam have been observed earlier. ${ }^{9,10}$ However, when the speed of rotation of the polarization was increased beyond $15 \mathrm{rpm}$ by use of a rotating half wave plate, the RBC (trapped at $120 \mathrm{~mW}$ ) was found not to follow the rotating polarization. Thus, the alignment torque due to linear polarization is limited by the power of the trapping beam that can be incident on the RBC. It may be noted that some biological materials can have sufficient optical birefringence to cause orientation/rotation in polarized light. ${ }^{11}$

In summary, we have presented the application of confocal microscopy for 3-D imaging of RBCs oriented by a trapping beam. Under different osmolarities of the buffer, the threedimensionally reconstructed images showed that the trapped RBC maintains its shape and is oriented in the vertical direction. Further, under both isotonic and hypertonic situations, the vertically oriented discotic $\mathrm{RBC}$ is aligned along the plane of polarization of the trapping beam. Using dual optical tweezers, the RBC could also be oriented three-dimensionally in a controlled manner. Since no folding of the RBC was observed under the optical trapping beam, the rotational mechanism based on optical birefringence caused by folding of the RBC was ruled out. The alignment and rotation of the RBC with polarization of the tweezers' beam can rather be attributed to its form-birefringence.

\section{References}

1. S. K. Mohanty, A. Uppal, and P. K. Gupta, "Self-rotation of red blood cells in optical tweezers: prospects for high throughput malaria diagnosis," Biotechnol. Lett. 26, 971-974 (2004); "Self-rotation of red blood cells in optical tweezers: prospects for high throughputmalaria diagnosis," see also Opt. Photonics News 15(12), 19 (2004).

2. S. K. Mohanty, K. S. Mohanty, and P. K. Gupta, "RBCs under optical tweezers as cellular motors and rockers: microfluidic applications," Proc. SPIE 6326, 63262E, (2006).

3. A. La Porta and M. D. Wang, "Optical torque wrench: angular trapping, rotation, and torque detection of quartz microparticles," Phys. Rev. Lett. 92, 190801 (2004).

4. J. A. Dharmadhikari, S. Roy, A. K. Dharmadhikari, S. Sharma, and D. Mathur, "Naturally occurring, optically driven, cellular rotor," Appl. Phys. Lett. 85, 6048-6050 (2004).

5. K. O. Greulich, Micromanipulation by Light in Biology and Medicine: The Laser Microbeam and Optical Tweezers, p. 237 Birkhäuser, Basel (1999).

6. S. K. Mohanty, K. S. Mohanty, and P. K. Gupta, "Dynamics of interaction of RBC with optical tweezers," Opt. Express 13, 4745 (2005), also in "Dynamics of interaction of RBC with optical tweezers," V. J. Biol. Phys. Res. (Oct. 1 2005).

7. A. Hoffmann, G. Meyer zu Hörste, G. Pilarczyk, S. Monajembashi, V. Uhl, and K. O. Greulich, "Optical tweezers for confocal microscopy," Appl. Phys. B: Lasers Opt. 71, 747-753 (2000).

8. M. E. J. Friese, T. A. Nieminen, N. R. Heckenberg, and N. R. Rubinsztein-Dunlop, "Optical alignment and spinning of lasertrapped microscopic particles," Nature 394, 348-350 (1998).

9. P. Galajda and P. Ormos, "Orientation of flat particles in optical tweezers by linearly polarized light," Opt. Express 11, 446-451 (2003).

10. S. Bayoudh, T. A. Nieminen, N. R. Heckenberg, and H. RubinszteinDunlop, "Orientation of biological cells using plane-polarized Gaussian beam optical tweezers," J. Mod. Opt. 50, 1581-1590 (2003).

11. G. Garab, P. Galajda, I. Pomozi, L. Finzi, T. Praznovszky, P. Ormos, and $\mathrm{H}$. van Amerongen, "Alignment of biological microparticles by a polarized laser beam," Eur. Biophys. J. 34, 335-341 (2005). 